\title{
Awake minimally invasive surgery as a game changer in lung cancer
}

\author{
Hussein Elkhayat ${ }^{1}$, Diego Gonzalez Rivas ${ }^{2,3}$ \\ ${ }^{1}$ Cardiothoracic Surgery, Faculty of Medicine, Assiut University, Assiut 71526, Egypt. \\ ${ }^{2}$ Department of Thoracic Surgery and Minimally Invasive Thoracic Surgery Unit (UCTMI), Coruña University Hospital, Coruña \\ 15006, Spain. \\ ${ }^{3}$ Department of Thoracic Surgery, Shanghai Pulmonary Hospital, Tongji University School of Medicine, Shanghai 200433, China.
}

Correspondence to: Prof. Hussein Elkhayat, Cardiothoracic Surgery, Assiut University Hospitals, Assiut 71526, Egypt.

E-mail:dr_khayat@hotmail.com

How to cite this article: Elkhayat $\mathrm{H}$, Gonzalez Rivas D. Awake minimal invasive surgery as a game changer in lung cancer. Miniinvasive Surg 2020;4:85. http://dx.doi.org/10.20517/2574-1225.2020.76

Received: 28 Jul 2020 First Decision: 21 Aug 2020 Revised: 25 Sep 2020 Accepted: 14 Oct 2020 Published: 5 Dec 2020

Academic Editor: Marcelo F. Jimenez Copy Editor: Cai-Hong Wang Production Editor: Jing Yu

\begin{abstract}
Surgery still offers the best option for patients with early stage non-small cell lung cancer that can tolerate surgery. With the increase in screening programs, more patients are diagnosed at early stages of cancer. Sadly, not all of them are fit for surgery, but with minimally invasive approaches, large number of those patients can be offered surgery and get a better overall survival. Awake non-intubated video assisted thoracic surgery resection is one of the most recent technique that we believe to be a game changer in this spectrum of patients who were previously classified as medically inoperable.
\end{abstract}

Keywords: Video assisted thoracic surgery, stereotactic body radiotherapy, early stage, non-small cell lung cancer, minimally invasive, inoperable

\section{INTRODUCTION}

With an increase in the number of patients diagnosed with early stage lung cancer, options for cure include surgery and stereotactic body radiotherapy (SBRT). Recent guidelines published from ASTRO recommend the use of SBRT in cases that medically inoperable ${ }^{[1]}$. These results were augmented by the most recent publication that found that median survival was significantly greater after surgery compared to SBRT in a risk-adjusted matched cohort of patients judged to be surgical candidates. The authors recommend

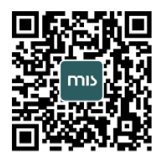


that every operable patient considering primary SBRT should be educated regarding this difference in survival ${ }^{[2]}$. Even though this was a retrospective study, it makes a real clinical trial to compare both maneuvers very difficult. Such a trial that will contain two very different patient populations, one group will be fit for surgery and the other one is unfit, with a significant bias regarding long-term outcomes and overall survival. Surgery still offers the best option for patients that can tolerate it ${ }^{[3]}$. With the increase in minimally invasive approaches, more patients can be offered surgery and achieve better overall survival. Awake non-intubated video assisted thoracic surgery (VATS) resection is one of the most recent technique that we believe to be a game changer in this spectrum of patients who were previously classified as medically inoperable.

\section{CHALLENGES WITH EARLY STAGE LUNG CANCER}

As more screening programs become readily available for lung cancer, more patients are diagnosed with early stage lung cancer ${ }^{[4]}$. Most of these patients have very mild or no symptoms, making it troublesome for physicians to ask them to consider high risk or very complex interventions. However, at the same time, these patients mostly have comorbidities that increase with age such as cardiovascular problems and limited pulmonary reserves, and even if we can offer them curative surgery, a large percentage of them are medically inoperable. There was no specific definition of such inoperability. With an average age of diagnosis of 70 years $^{[5]}$, lung cancer patients often have a level of baseline frailty, along with concomitant comorbid conditions, especially those associated with risk factors for non-small cell lung cancer such as heart disease, chronic obstructive pulmonary disease, and loss of pulmonary parenchyma. Age is not the sole factor to determine medical operability, several factors such as performance status, presence of medical comorbidities, and pulmonary function tests, contribute to overall risk assessment.

With this above-mentioned status, surgeons try to improve the overall perioperative experience and facilitate surgery for more lung cancer patients by moving from open surgery to less invasive surgery. In thoracic surgery, there was a rapid pace of change from open thoracotomy to multiport VATS, uniportal VATS, and subxiphoid VATS, all aiming at decreasing the surgical burden on the patients by decreasing the incision and limited access surgery. Yet, there was another important factor that attributed to mortality and morbidity and that was anesthesia. Since the development of minimally invasive lung resection, almost all cases were operated under general anesthesia with double lumen endotracheal intubation. Tracheobronchial injuries ${ }^{[6-8]}$, prolonged effect of neuromuscular blockers ${ }^{[\rho]}$, and pulmonary complications postoperatively ${ }^{[10]}$ are all possible complications of general anesthesia and double lumen endotracheal intubation. This drove surgeons to think of non-intubated VATS as a way to avoid these complications and improve the patients' overall experience. This seems to have an extra advantage in patients with impaired pulmonary function who are usually unfit for general anesthesia and will typically be deferred to another therapeutic option inferior to radical curative surgery.

Another challenge appears on the surface is the ground glass opacities (GGOs) which become more detected nowadays thanks to screening programs. Incidence of cancer in GGO has been reported as high as $63 \%$ so most surgeons prefer to get a biopsy before resection, but some prefer direct surgical resection. This can be possible for peripheral lesions, but for central GGOs it is very challenging to obtain a preoperative pathology. Hence, a minimally invasive approach can offer both diagnostic and therapeutic solutions that cannot be done with the SBRT approach ${ }^{[1]}$. Even in peripheral GGOs, localization in non-collapsed lung or emphysematous patients is limited especially with coughing and movements of the diaphragm and the mediastinum in case of awake VATS. This can be overcome by intrathoracic vagal and phrenic nerve blocks or administration of aerosolized lidocaine. For nodules and GGOs not amenable to finger palpation, preoperative CT guided hook wire insertion or a preoperative CT-guided dye localization can improve the intraoperative localization and shortened the operative time and manipulation ${ }^{[12]}$. 


\section{AWAKE VATS: WHAT CAN BE OFFERED}

Surprisingly, awake thoracic surgery is not a brand-new intervention. The idea dated back to 1923 to Eloesser ${ }^{[13]}$ who, in his report in California State Journal of Medicine, stated that, "....almost all operations upon the bony thorax should be done under local and regional anesthesia..." With the improvements in general anesthesia and tracheal intubation, regional and local anesthesia vanished gradually ${ }^{[14]}$ till the evolution of VATS in the early 1990s when more surgeons showed increased interest in keeping the patients breathing spontaneously with some degree of awareness to avoid the problems associated with general anesthesia and double lumen endotracheal intubation ${ }^{[15]}$. Later, surgeons started to look for the postoperative lymphocyte responses and other immune responses in cases of awake versus conventional general anesthesia, and they demonstrated attenuated stress response after awake VATS in comparison to an equivalent procedure performed under general anesthesia and one-lung ventilation ${ }^{[16,17]}$. This in turn attracted more surgeons to adopt this technique in cancer surgery as lower immune system responses are highly desirable in those patients. In 2014, Gonzalez-Rivas ${ }^{[18]}$ published the first report of awake uniportal VATS lobectomy. Two years later, the Tor Vergata group published the first series of 1000 patients operated using awake uniportal VATS ${ }^{[19]}$. Moreover, the development of uniportal VATS in conjunction with awake surgery helped to perform some operations in the outpatient setting ${ }^{[20]}$. Non-intubated VATS can be nonintubated with deep sedation in which patients are relaxed with good airway control via facemask, nasal cannula, or even laryngeal masks. This type awake VATS is suitable for major resections with longer operative time. The second type is loco-regional anesthesia in awake patients and this is commonly used for diagnostic VATS and minor resections ${ }^{[21,2]}$.

\section{FUTURE PROSPECTIVE}

This contentious effort drove more surgeons to use this technique in more frail and high risk surgery patients. Wang et al. ${ }^{[3]}$, in Taiwan, retrospectively investigated the results of 28 patients with impaired lung function (preoperative forced expiratory volume in 1 second $<70 \%$ of the predicted value) who underwent non-intubated VATS. Only eighteen patients in this series had primary lung cancers, of those patients, lobectomy was performed in 4 , segmentectomy in 3, and wedge resection in 11, with lymph node sampling adequate for staging. Wang et al. ${ }^{[3]}$ reported no mortalities after 30 days; however, conversion to tracheal intubation and one-lung ventilation was required in one patient, an 80-year-old man, because of persistent intraoperative wheezing and labored breathing. The intubation was performed smoothly in the lateral position. He required ventilator support postoperatively but was weaned off the next morning. No patients required conversion to a thoracotomy or blood component therapy. This report gives hope that some patients, previously categorized with impaired pulmonary function and medically inoperable patients, can now safely be offered a curative surgery. Yet, sharp cardiac and pulmonary indications for awake non-intubated surgery have not been fully investigated and are still under trials to determine the exact cut-off values. The most accepted contraindications so far for awake or non-intubated uniportal VATS can be categorized into: (1) patient related (i.e., obesity, neurological conditions, uncontrolled gastroesophageal regurgitation, central hypoventilation syndrome, persistent cough or mucus retention, and hemodynamically instable or severely hypoxia/hypercapnia); (2) anesthesiologist-related (i.e., difficult intubation, technical contraindications to general anesthesia, need to protect the contralateral lung from spillage of endobronchial contents, and inexperienced or non-cooperative team); and (3) surgeon-related (i.e., uniportal VATS experience and previous operations with adhesions ${ }^{[23]}$.

Khorfan et al. ${ }^{[2]}$ retrospectively investigate records from 2004 to 2016 of patients who were fit for surgery, but refused to have surgery and preferred SBRT. From 138,143 patients who met our inclusion criteria, they found that 1359 patients $(0.98 \%)$ refused recommended surgery and elected SBRT. Numbers are increasing every year. Propensity matching resulted in 1,315 well-balanced pairs. Surgery was associated with higher median survival $(74$ months $v s .47$ months; $P<0.01$ ) in the matched cohort. Survival benefit persisted 
after adjusting for covariates on Cox regression (hazard ratio, 1.69; $P<0.01$ ). The authors concluded that, operable patients considering primary SBRT should be educated regarding this difference in survival. Even though they reported that in the surgery group, 102,596 (75.0\%) underwent lobectomy and 25,048 (18.3\%) sub-lobar resection with the remaining patients undergoing other or unspecified types of resection, they did not report how many patients who refuse surgery were offered minimally invasive surgery, or whether they were offered lobectomy or sub-lobar resection and whether this was a point of refusal for surgical option or not. According to Sihoe ${ }^{[24]}$, for any new technique or procedure, the evolution of research and evidence-gathering can be categorized into five distinct phases that neatly correspond to the development of a human. Awake uniportal VATS must go to from Infancy-safety \& feasibility and Childhood-crude benefit to the next level of technique maturity in this stage which is Adolescence-objective, quantifiable benefit then to Adulthood-treatment efficacy and finally to Maturity-sustainability.

\section{CONCLUSION}

Although there is cumulative evidence of the safety and feasibility of awake uniportal VATS lung resection for early stage lung cancer and the preliminary results suggesting benefits for patients with limited pulmonary functions, more prospective studies are needed to investigate long-term outcome on patients who have limited pulmonary reserve and increased frailty.

\section{DECLARATIONS}

\section{Authors' contributions}

Collection and assembly of data, data analysis and interpretation: Elkhayat $\mathrm{H}$

Manuscript writing: Elkhayat H, Gonzalez Rivas D

Conception and design, administrative support, provision of study material: Gonzalez Rivas D

\section{Availability of data and materials}

Not applicable.

\section{Financial support and sponsorship}

None.

\section{Conflicts of interest}

All authors declared that there are no conflicts of interest.

\section{Ethical approval and consent to participate}

Not applicable.

\section{Consent for publication}

Not applicable.

\section{Copyright}

(c) The Author(s) 2020

\section{REFERENCES}

1. Videtic GMM, Donington J, Giuliani M, Heinzerling J, Karas TZ, et al. Stereotactic body radiation therapy for early-stage non-small cell lung cancer: executive summary of an ASTRO evidence-based guideline. Pract Radiat Oncol 2017;7:295-301.

2. Khorfan R, Kruser TJ, Coughlin JM, Bharat A, Bilimoria KY, et al. Survival of primary stereotactic body radiation therapy compared with surgery for operable stage I/II non-small cell lung cancer. Ann Thorac Surg 2020;110:228-34.

3. Wang ML, Hung MH, Hsu HH, Chan KC, Cheng YJ, et al. Non-intubated thoracoscopic surgery for lung cancer in patients with impaired pulmonary function. Ann Transl Med 2019;7:40. 
4. Detterbeck FC, Mazzone PJ, Naidich DP, Bach PB. Screening for lung cancer: Diagnosis and management of lung cancer, 3rd ed: American College of Chest Physicians evidence-based clinical practice guidelines. Chest 2013;143:e78S-92.

5. Howlader N, Noone AM, Krapcho M, Miller D, Brest A, et al. SEER cancer statistics review, 1975-2016. National Cancer Institute. Bethesda, MD; 2019. pp. 1423-37. Available from: https://seer.cancer.gov/archive/csr/1975_2016/. [Last accessed on 21 Oct 2020]

6. Liu S, Mao Y, Qiu P, Faridovich KA, Dong Y. Airway rupture caused by double-lumen tubes: a review of 187 cases. Anesth Analg 2020; doi: 10.1213/ANE.0000000000004669.

7. Fitzmaurice BG, Brodsky JB. Airway rupture from double-lumen tubes. J Cardiothorac Vasc Anesth 1999;13:322-9.

8. Brodsky JB, Shulman MS, Mark JB. Airway rupture with a disposable double-lumen tube. Anesthesiology 1986;64:415.

9. Murphy GS, Szokol JW, Avram MJ, Greenberg SB, Shear T, et al. Postoperative residual neuromuscular blockade is associated with impaired clinical recovery. Anesth Analg 2013;117:133-41.

10. Della Rocca G, Coccia C. Acute lung injury in thoracic surgery. Curr Opin Anaesthesiol 2013;26:40-6.

11. Migliore M, Fornito M, Palazzolo M, Criscione M, Gangemi M, et al. Ground glass opacities management in the lung cancer screening era. Ann Transl Med 2018;6:90.

12. Passera E, Rocco G. Awake video-assisted thoracic surgery resection of lung nodules. Video Assist Thorac Surg 2018;3.

13. Eloesser L. Local anesthesia in major surgery: its uses and limitations. Cal State J Med 1923;21:412-5.

14. Tamburrini A, Mineo TC. A glimpse of history: non-intubated thoracic surgery. Video Assist Thorac Surg 2017;2.

15. Pompeo E, Mineo D, Rogliani P, Sabato AF, Mineo TC. Feasibility and results of awake thoracoscopic resection of solitary pulmonary nodules. Ann Thorac Surg 2004;78:1761-8.

16. Vanni G, Tacconi F, Sellitri F, Ambrogi V, Mineo TC, et al. Impact of awake videothoracoscopic surgery on postoperative lymphocyte responses. Ann Thorac Surg 2010;90:973-8.

17. Tacconi F, Pompeo E, Sellitri F, Mineo TC. Surgical stress hormones response is reduced after awake videothoracoscopy. Interact Cardiovasc Thorac Surg 2010;10:666-71.

18. Gonzalez-Rivas D, Fernandez R, de la Torre M, Rodriguez JL, Fontan L, et al. Single-port thoracoscopic lobectomy in a nonintubated patient: the least invasive procedure for major lung resection? Interact Cardiovasc Thorac Surg 2014;19:552-5.

19. Mineo TC, Tamburrini A, Perroni G, Ambrogi V. 1000 cases of tubeless video-assisted thoracic surgery at the Rome Tor Vergata University. Future Oncol 2016;12:13-8.

20. Rocco G, Romano V, Accardo R, Tempesta A, La Manna C, et al. Awake single-access (uniportal) video-assisted thoracoscopic surgery for peripheral pulmonary nodules in a complete ambulatory setting. Ann Thorac Surg 2010;89:1625-7.

21. Migliore M. Efficacy and safety of single-trocar technique for minimally invasive surgery of the chest in the treatment of noncomplex pleural disease. J Thorac Cardiovasc Surg 2003;126:1618-23.

22. Rocco G. Non-intubated uniportal lung surgerydagger. Eur J Cardiothorac Surg 2016;49 Suppl 1:i3-5.

23. Gonzalez-Rivas D, Bonome C, Fieira E, Aymerich H, Fernandez R, et al. Non-intubated video-assisted thoracoscopic lung resections: the future of thoracic surgery? Eur J Cardiothorac Surg 2016;49:721-31.

24. Sihoe AD. Reasons not to perform uniportal VATS lobectomy. J Thorac Dis 2016;8:S333-43. 